\title{
Sensor Based on Multiple Fiber Bragg Gratings for Diameter Measurement
}

\author{
Victor Cardoso ${ }^{1,4,7}$, Paulo Caldas ${ }^{4,5}$, M. Thereza R. Giraldi ${ }^{2}$, Orlando Frazão ${ }^{3,4}$, Claudio Carvalho ${ }^{6}$, João Costa ${ }^{1}$, and \\ José L. Santos 3,4 \\ ${ }^{1}$ Federal University of Pará, Applied Electromagnetism Laboratory, Rua Augusto Corrêa, 01, 66075-110, Belém, Pará, Brazil \\ ${ }^{2}$ Military Institute of Engineering, Laboratory of Photonics, Praça Gen. Tibúrcio, 80,22290-270, Rio de Janeiro, Brazil \\ ${ }^{3}$ Department of Physics and Astronomy, Faculty of Sciences of University of Porto, Rua do Campo Alegre, 687, 4169-007 Porto, \\ Portugal \\ ${ }^{4}$ Institute for Systems and Computer Engineering, Technology and Science, Rua do Campo Alegre, 687, 4169-007 Porto, Portugal \\ ${ }^{5}$ Polytechnic Institute of Viana do Castelo, Rua Escola Industrial e Comercial de Nun’Álvares, 4900-347, Viana do Castelo, Portugal \\ ${ }^{6}$ Brazilian Agricultural Research Corporation \\ ${ }^{7}$ victorcard@ufpa.br
}

\begin{abstract}
A method based on multiple Fiber Bragg Gratings (FBG) and a structure fabricated using a 3D printer for diameter measurement is proposed and experimentally demonstrated. The sensor is easily developed inserting the FBG on the structure and fixing the FBG in two points. Due to this arrangement, when the diameter is reduced a force is applied on the sensor and this causes a wavelength shift. The study is divided in two steps using one experimental setup. At first, each FBG response is independently evaluated concerning the diameter variation and after that, the FBGs are evaluated together. The results demonstrated that the structure can be used as a way to monitor the diameter variation in some applications.
\end{abstract}

\section{Introduction}

In several areas the diameter monitoring presents an important function because it will influence the production and consequently generate financial losses and in some cases can also lead to social problems. For example, in the gaseous hydrogen transportation infrastructure the pressure into the pipeline is high, causing deformation and/or fatigue and consequently a disruption [1].Another example is the expansion and contraction monitoring of the tree stem that is directly related to irrigation, since it depends on the soil water deficit [2-4]. The use of optical sensors for these cases is interesting because they present characteristics such as immunity to electromagnetic interference, compact size, resistance to pressure, heat and corrosion, and the possibility of transmission over long distances. The Fiber Bragg Grating (FBG) is used extensively in several applications, mainly, for strain measurement since this parameter is associated with the change in the wavelength of the reflected signal, which is accurately determined and catalogued for each grating [5].

In this work, we investigate a method for diameter monitoring using a sensor based on multiple Fiber Bragg Gratings. We use an experimental setup that uses the principle of the strain gauge based on FBG for diameter monitoring. We develop a structure in a $3 \mathrm{D}$ printer so that it was possible to perform the analysis of the diameter variation of different structures. This work presents preliminary results using only two FBGs

\footnotetext{
* This study was financed in part by the Coordenação de Aperfeiçoamento de Pessoal de Nível Superior - Brasil (CAPES) - Finance code 001, the Conselho Nacional de Desenvolvimento Cientifico e Tecnologico $(\mathrm{CNPq})$, Institute for Systems and Computer Engineering, Technology and Science (INESC TEC) and the Department of Physics and Astronomy, Faculty of Sciences of University of Porto from Portugal)
}

\section{Preliminary Experimental Setup and Sensor Operation}

We proposed a configuration that can be observed in Fig. 1(a). We developed the structures using a 3D printer for the FBGs supporting and to simulate the diameter to be monitored, as shown in Fig. 1(b). We used two pieces for this experiment, one for each FBG. The diameter variation is made using a linear moving translation stage. The FBGs were fixed in the piece in two points so that when the diameter changes a force will tension or compress the sensor, as shown Fig. 1(b). The FBGs were used in series. Light from a broadband source was injected in the circulator and after crossing it, the optical beam was introduced in the sensing head formed by the two FBGs. The reflection spectrum of the sensing head was monitored with an Optical Spectrum Analyzer (Yokogame AQ6370C), which is illustrated in Fig. 1(a). The FBGs were fabricated in single-mode fiber with a reflection wavelength of $1558 \mathrm{~nm}$ each one. The pieces were created so that it was possible to do an analysis of circular structures like a pipeline, the stem of a tree and other structures which need to have their diameter variation monitored. A strain is applied on the optical fiber by the variation of the diameter of the structure $(\Delta d)$ and therefore it was possible to obtain the sensor response. In order to obtain the sensitivity of the sensors, in this experiment we analyzed each FBG separately and after that the FBGs were tested together.

\section{Results and Discussion}

The results obtained from the experiments are depicted in Fig. 2. It is possible to observe that when reducing the 


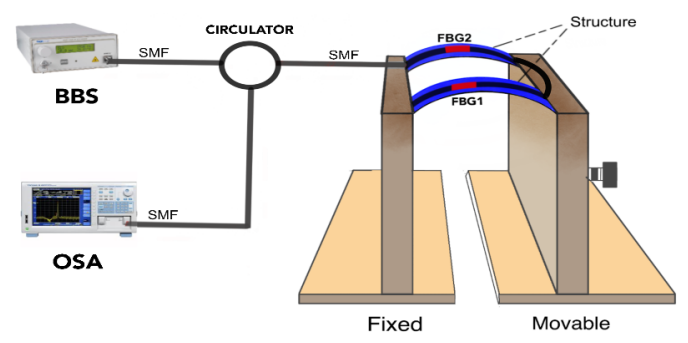

(a)

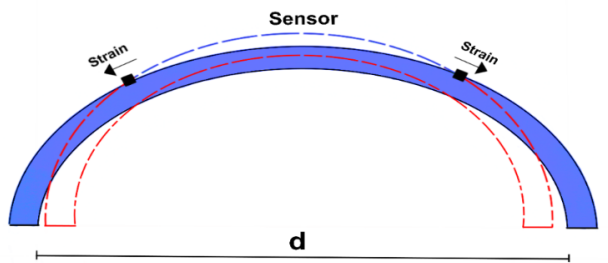

(b)

Figure 1. (a) Schematic of the experimental setup. (b) Mechanism of operation of the structure that demonstrates the diameter reduction using the proposed structure developed with a $3 \mathrm{D}$ Printer.

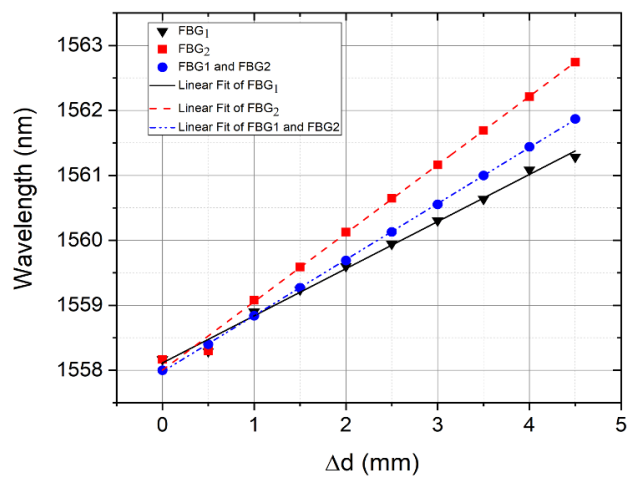

Figure 2. Wavelength shift caused by the diameter reduction.

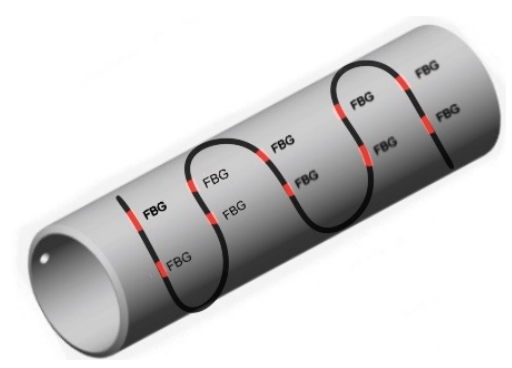

Figure 3. Schematic of the experimental support under construction for Multiple FBGs in a cylindrical structure. diameter, the results presented a linear behavior when using just one FBG or both of them. When just one FBG is under test, it presented a wavelength shift due to the applied force, while the other one remained centered at 1558 $\mathrm{nm}$ since it was not tensioned. For this case, FBG1 and FBG2 presented good linear responses with $R^{2}=0.9940$ and $R^{2}=0.9959$, respectively. The sensitivity obtained was $0.7261 \mathrm{~nm} / \mathrm{mm}$ and $1.05 \mathrm{~nm} / \mathrm{mm}$, respectively.

When we analyzed the FBGs acting together, the same force was applied in each piece. It was possible to observed a wavelength shift due to the two FBGs together, superimposed. For this case, the sensor presented a good linear response with $R^{2}=0.9994$ and a sensitivity of the $0.8577 \mathrm{~nm} / \mathrm{mm}$. This study presents preliminary results for two FBGs. The next step will be to perform measurements on cylindrical structures using multiple FBGs, as shown in Fig. 3, applying non-uniform forces and consequently variations in the cylindrical structure. Therefore, it will be possible to measure the eccentricity variation around and/or along the cylindrical structure.

\section{Conclusion}

In conclusion, we proposed an experimental investigation on a method for diameter monitoring using a sensor based on multiple Fiber Bragg Gratings. We developed an experimental setup that is simple and repeatable. The results using the proposed method demonstrate that it is possible to monitor the diameter of some applications such as pipelines, expansion and contraction of the tree stem, Structure Health Monitoring (SHM) and so on. The use of multiple FBGs enable to measure the eccentricity variation around and/or along a cylindrical structure. With the printed piece used in this work, it is possible to use the FBG to monitor the diameter variation from 0 to $4.5 \mathrm{~mm}$. Diameter variations of these sizes in some stem trees permit to obtain the irrigation levels of these vegetations. This study presents preliminary results and the next step will be to perform measurements on cylindrical structures using multiple FBGs.

\section{References}

[1] R.L. Amaro, E.S. Drexler, A.J. Slifka, International Journal of Fatigue 62, 249 (2014)

[2] M. Cohen, D.A. Goldhamer, E. Fereres, J. Girona, M. Mata, The Journal of Horticultural Science and Biotechnology pp. 55-60 (2001)

[3] M. Martín-Palomo, M. Corell, I. Girón, L. Andreu, E. Trigo, Y. López-Moreno, A. Torrecillas, A. Centeno, D. Pérez-López, A. Moriana, Agricultural Water Management 218, 115 (2019)

[4] R. Çakir, Field Crops Research (2004)

[5] H. lin Liu, Z. wei Zhu, Y. Zheng, B. Liu, F. Xiao, Optical Fiber Technology (2018) 